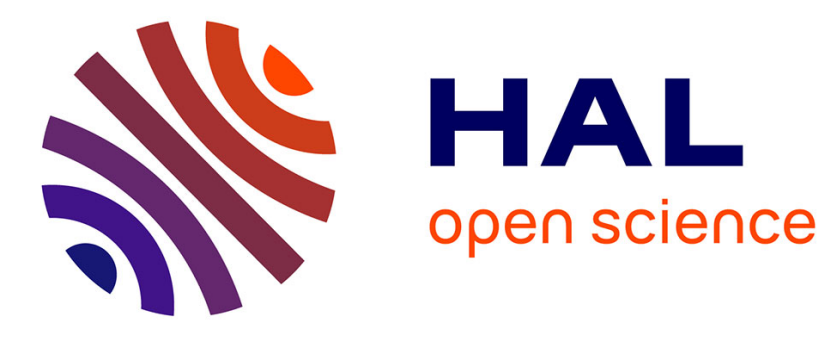

\title{
Étudiants africains : des émigrés comme les autres
}

Hugo Bréant

\section{To cite this version:}

Hugo Bréant. Étudiants africains: des émigrés comme les autres: Sélectivité sociale du visa et (im)mobilités spatiales des étudiants internationaux comoriens et togolais. Politix, 2018, 3 (123), pp.195-218. 10.3917/pox.123.0195 . hal-02025354

\section{HAL Id: hal-02025354 \\ https://hal-normandie-univ.archives-ouvertes.fr/hal-02025354}

Submitted on 31 Mar 2021

HAL is a multi-disciplinary open access archive for the deposit and dissemination of scientific research documents, whether they are published or not. The documents may come from teaching and research institutions in France or abroad, or from public or private research centers.
L'archive ouverte pluridisciplinaire $\mathbf{H A L}$, est destinée au dépôt et à la diffusion de documents scientifiques de niveau recherche, publiés ou non, émanant des établissements d'enseignement et de recherche français ou étrangers, des laboratoires publics ou privés. 


\section{ÉTUDIANTS AFRICAINS : DES ÉMIGRÉS COMME LES AUTRES Sélectivité sociale du visa et (im)mobilités spatiales des étudiants internationaux comoriens et togolais Hugo Bréant}

De Boeck Supérieur | « Politix»

2018/3 n 123 | pages 195 à 218

ISSN 0295-2319

ISBN 9782807392021

Article disponible en ligne à l'adresse :

https://www.cairn.info/revue-politix-2018-3-page-195.htm

Distribution électronique Cairn.info pour De Boeck Supérieur.

(C) De Boeck Supérieur. Tous droits réservés pour tous pays.

La reproduction ou représentation de cet article, notamment par photocopie, n'est autorisée que dans les limites des conditions générales d'utilisation du site ou, le cas échéant, des conditions générales de la licence souscrite par votre établissement. Toute autre reproduction ou représentation, en tout ou partie, sous quelque forme et de quelque manière que ce soit, est interdite sauf accord préalable et écrit de l'éditeur, en dehors des cas prévus par la législation en vigueur en France. Il est précisé que son stockage dans une base de données est également interdit. 


\title{
Étudiants africains : des émigrés comme les autres
}

\section{Sélectivité sociale du visa et (im)mobilités spatiales des étudiants internationaux comoriens et togolais}

Hugo BréANt

\begin{abstract}
Résumé - S'ils sont associés à des discours politiques soulignant la forte attractivité de la France, les étudiants étrangers demeurent des étrangers comme les autres, progressivement soumis à des politiques de contrôle de leur entrée et de leur séjour. Les étudiants africains sont en première ligne de ce processus de fermeture des frontières, à l'œuvre dès les consulats de France. Cet article propose de renverser la perspective en observant, non pas la production de la politique des visas, mais les perceptions et les pratiques des étudiants qui l'affrontent. Enquêter les parcours d'étudiants qui ont obtenu ou se sont vus refuser leur visa permet de saisir les logiques des inégalités qui persistent face à ce filtre consulaire socialement très sélectif. Souvent captifs d'une procédure du visa étudiant diversement appropriée, les étudiants développent différentes stratégies pour s'accommoder de la frontière, ou la contourner, selon leurs propriétés sociales, leurs histoires familiales et leurs ressources individuelles et collectives. Cette approche de l'action publique par le bas, et par ses usagers, permet de saisir comment l'inextricable enchevêtrement des frontières, à la fois politiques et sociales, renforce l'immobilité spatiale des uns, mais surtout complexifie les mobilités des autres.
\end{abstract}

Mots clés - mobilité internationale, étudiants, frontières, visas, inégalités sociales, Comores, Togo 


\section{Introduction}

Depuis trois décennies, la sociologie politique a développé des outils à même de "saisir l'action publique par le bas ${ }^{1}$ ", c'est-à-dire à hauteur des agents qui produisent les dispositifs politiques, mais aussi des rapports que les usagers entretiennent à ces administrations. Ces travaux ont souligné à la fois les mises en œuvre localement contrastées des politiques nationales, les (dés)ajustements entre les trajectoires personnelles des agents et les missions qui leur sont assignées, ou encore les pratiques souvent discrétionnaires de ces derniers. Ces recherches se sont particulièrement attachées à rendre compte des formes de domination qui s'exercent sur des usagers captifs des "prescriptions identitaires $^{2} »$ qu'on leur adresse. Ces travaux ont ainsi mis au jour la violence symbolique à l'œuvre dans le face-à-face entre des agents souvent peu formés et isolés, et des usagers placés en position de « demander l'aumône ${ }^{3}$ ». En s'appuyant sur le cas spécifique d'étudiants africains qui demandent leur visa dans un consulat français, cet article propose un prolongement de ces travaux en suivant l'appel à se tourner davantage vers les usagers ${ }^{4}$. Il s'agira de mieux comprendre les effets des politiques de contrôle des mobilités internationales sur les parcours individuels. Ces procédures d'obtention d'un visa étudiant peuvent en effet parfois être vécues comme de simples formalités administratives ou souvent comme des obstacles difficilement surmontables. Mais elles exercent toujours des effets bien plus durables sur les trajectoires biographiques et sur les histoires familiales de ces étudiants. En mettant en regard les logiques d'exclusion politique et les propriétés des individus qui s'y confrontent, il devient possible de mieux saisir comment les frontières politiques et les frontières sociales s'enchevêtrent pour produire des (im)mobilités spatiales.

\section{Étudier sans frontière?}

L'approche de l'action publique par le bas a été particulièrement suivie dans le cas du contrôle des populations migrantes, qu'il s'agisse des consulats auxquels se confrontent les candidats à l'émigration, des préfectures fréquentées par les immigrés désireux de régulariser leur situation administrative ${ }^{5}$, ou des services de l'État chargés de décider de l'attribution de la nationalité française

1. Lascoumes (P.), Le Galès (P.), Sociologie de l'action publique, Paris, Armand Colin, 2012.

2. Dubois (V.), La vie au guichet. Relation administrative et administration de la misère, Paris, Economica, 2008.

3. Siblot (Y.), Faire valoir ses droits au quotidien. Les services publics dans les quartiers populaires, Paris, Presses de Sciences Po, 2006.

4. Avril (C.) et al., «Les rapports aux services publics des usagers et agents de milieux populaires : quels effets des reformes de modernisation ? ", Sociétés contemporaines, 58 (2), 2005.; Warin (P.), « Les relations de service, objet de recherches en sciences sociales ", Pyramides, 7, 2003.

5. Spire (A.), Accueillir ou reconduire. Enquête sur les guichets de l'immigration, Paris, Raisons d'agir, 2007. 
à des étrangers ${ }^{6}$. Par les «savoirs institués " et les «savoirs pratiques" qu'ils mettent en œuvre, les agents de ces institutions contribuent à hiérarchiser les demandes et, ce faisant, à construire et à redéfinir la frontière légale ${ }^{7}$. Ce pouvoir discrétionnaire d'appréciation des dossiers individuels se fonde sur une politique du soupçon qu'alimente la figure de l'overstayer, c'est-à-dire du migrant qui entre légalement sur le territoire mais souhaite s'y maintenir plus longtemps qu'il n'y est autorisé.

Seules les mobilités étudiantes semblent a priori jouir d'une relative bienveillance et passer sous le radar des régulations politiques. En effet, en accordant chaque année entre 70 et 90000 visas à des étudiants, la France est le quatrième pays d'accueil des 4,3 millions d'étudiants mobiles à l'échelle internationale ${ }^{8}$. Des frottements existent pourtant entre les engagements gouvernementaux à renforcer l'attractivité internationale de la France et les politiques inhospitalières régulièrement adoptées à l'encontre des étudiants étrangers ${ }^{9}$. Dès 1979, le gouvernement instaurait une série de conditions à leur entrée et leur séjour sur le territoire (préinscription universitaire, test de langue, conditions de ressources, engagement à repartir au terme des études) ${ }^{10}$. En 2007, le ministère de l'Immigration recevait pour consigne de ne favoriser que les entrées d'étudiants étrangers qui «répondent aux besoins économiques» du pays ${ }^{11}$. En 2011, la circulaire Guéant poursuivait ce mouvement de clôture, en cherchant à diminuer la délivrance des visas étudiants ${ }^{12}$ et en demandant aux préfets de complexifier encore le passage du statut d'étudiant au statut de travailleur ${ }^{13}$. En novembre 2018, Édouard Philippe maintient la volonté d'accueillir plus de

6. Mazouz (S.), La République et ses autres. Politiques de l'altérité dans la France des années 2000, Lyon, ENS Éditions, 2017.

7. Brabandt (H.), Mau (S.), «Regulating Territorial Access in a Globalized World. Visa-Waiver Policies in the USA and Austria ", Journal of Borderlands Studies, 28 (3), 2013 ; Infantino (F.), "La frontière au guichet », Champ Pénal, 7, 2010.; Infantino (F.), Rea (A.), «La mobilisation d'un savoir pratique local. Attribution des visas Schengen au Consulat Général de Belgique à Casablanca », Sociologies pratiques, 24 (1), 2012.; Maybritt (J.-A.), Spire (A.), « Dealing with Law in Migration Control: The Powers of Street-level Bureaucrats at French Consulates », Social Legal Studies, 23 (2), 2014 ; Satzewich (V.), Points of entry. How Canada's Immigration Officers Decide Who Gets In, Vancouver, Toronto, University of British Columbia Press, 2015.

8. Derrière les États-Unis, le Royaume-Uni et l'Australie, ce qui représente 310000 étudiants vivant en France en 2016. Sauf mention contraire, les données chiffrées proviennent des statistiques produites et publiées par l'agence Campus France.

9. Borgogno (V.), Streiff-Fénart (J.), «L'accueil des étudiants étrangers en France : politiques et enjeux actuels », Cahiers de l'URMIS, 5, 1999.

10. Slama (S.), La fin de l'étudiant étranger, Paris, L'Harmattan, 1999.

11. Wagner (A.-C.), «Attirer les talents internationaux. Les ambiguïtés d'une hospitalité sélective », Savoir/ Agir, 36 (2), 2016.

12. Après un léger reflux en 2011-2012, le taux de visas annuellement acceptés se maintient autour de $82-85 \%$.

13. Malgré son abrogation en 2012, la nature de l'emploi proposé aux étudiants peut encore leur être opposée et l'obtention d'une promesse d'embauche et du paiement par l'employeur d'une taxe liée au recrutement d'un salarié étranger sont toujours nécessaires. GISTI, «Étudiant.e.s., tri sélectif », Plein Droit, 91, 2011. 
500000 étudiants étrangers d'ici 2027, tout en instaurant une hausse drastique des frais d'inscription universitaire pour les étudiants extraeuropéens. Cette mesure s'inscrit dans un mouvement général de fermeture des frontières auquel n'échappent pas ces migrations « en col blanc ${ }^{14}$ ».

Dans l'ombre de ces politiques nationales, le rejet des étudiants étrangers est assuré par une sélection aux portes du territoire français. Depuis 2010, leurs demandes de visa étudiant sont encadrées par les agences Campus France. Interface entre les universités et les consulats, cette antenne aiguille les étudiants dans leur orientation et leur fournit une assistance matérielle dans la constitution de leurs dossiers. Campus France contrôle le dossier, émet un avis consultatif et le dépose au consulat, en lieu et place de l'étudiant. Cet avis se fonde sur trois critères centraux : le parcours scolaire, le projet d'études et les perspectives professionnelles visées au retour. Alexis Spire a bien montré les injonctions contradictoires qui guident le travail de cette nouvelle instance. En opérant de fait une présélection de plus en plus stricte des dossiers qui méritent d'être déposés, ces agences participent tacitement à la démultiplication « des filtres et des mécanismes de sélection » en amont du consulat ${ }^{15}$ et elles se transforment en un élément d'externalisation de la politique des visas étudiants.

Le contrôle politique des demandes de visas des étudiants internationaux est donc particulièrement ambigu, et répond à des logiques - méritocratique, utilitariste et sécuritaire - potentiellement contradictoires. L'excellence des parcours scolaires n'est encouragée que dans la mesure où la France peut tirer avantage de la présence durable de diplômés de certaines filières étudiantes et professionnelles. L'institutionnalisation de la gestion du visa étudiant a favorisé un alignement des mobilités étudiantes sur le contrôle général des flux migratoires. À cet égard, la situation des étudiants d'Afrique subsaharienne est éloquente. Sur les 354000 étudiants subsahariens qui étudiaient à l'étranger en 2016, la France en accueillait à elle seule près de $62000^{16}$, dont $41 \%$ d'étudiantes ${ }^{17}$. La délivrance des visas pour études, court ( 3 mois) et long séjour ( 12 mois), a connu une hausse constante, passant de 30000 visas annuels délivrés en 1998, à plus de 85000 en 2015. Pourtant, en 2006, le taux de refus de visa étudiant s'élevait à $20 \%$, contre $10 \%$ pour l'ensemble des demandes. Cette même année, «76,4 \% des refus de visas tous motifs confondus concernaient les visas étudiants ${ }^{18}$ ».

14. Geisser (V.), "Trop diplômés pour être honnêtes : la hogra des immigrés en "col blanc" ", Migrations Société, 138 (6), 2011.

15. Spire (A.), "Contrôler et choisir. La sélection des étudiants candidats à l'émigration vers la France ", in Mazzella (S.), dir., La mondialisation étudiante. Le Maghreb entre Nord et Sud, Paris, Karthala, 2009, p. 94.

16. L'Afrique subsaharienne, particulièrement francophone, est la deuxième zone d'origine de ces étudiants après le Maghreb.

17. Toutes régions d'origine confondues, les étudiantes étrangères sont en revanche majoritaires (53\%).

18. Réseau européen des migrations, Ministère de l'Intérieur, L’immigration des étudiants étrangers en France, Paris, 2012, p. 39.; Belaïsch (S.), Petersell (L.), dir., Visa refusé. Enquête sur les pratiques des consulats de France en matière de délivrance des visas. Rapport d'observation, Paris, La Cimade, 2010, p. 27. 
L'Afrique subsaharienne a été la première zone d'origine des étudiants confrontée à ces politiques restrictives. Dès 2001, les statistiques des visas accordés, des entrées sur le territoire et des effectifs étudiants ont commencé à diminuer ${ }^{19}$.

\section{"Mobiles " versus « immobiles involontaires ": deux catégories poreuses}

Les agents consulaires sont donc les « gardiens ${ }^{20}$ » d'une frontière légale qui fonctionne comme un filtre "à moitié perméable ", qui s'ouvre pour certains demandeurs et se referme en excluant les autres ${ }^{21}$. Mais ils ne construisent pas simplement une ligne de démarcation entre ceux qui remplissent les conditions légales du départ, et sont donc jugés aptes à émigrer, et ceux qui ne le peuvent pas, et deviennent des «immobiles involontaires ${ }^{22} »$. Si les politiques migratoires visent à distinguer migrations autorisées et clandestines, le travail consulaire opère un tri au sein même des demandes légales.

\section{Dispositif et conditions d'enquête}

Cet article s'appuie sur une recherche doctorale, menée entre 2010 et 2016, intitulée «Les chemins internationaux de la mobilité sociale », qui interroge les processus d'appropriation sociale des mobilités spatiales d'émigrés comoriens et togolais. L'enquête de terrain a permis de réaliser plus de 200 entretiens sociologiques, auprès d'émigrés et d'émigrés de retour, mais aussi de leurs proches, dans trois pays (France, Comores et Togo). Ces entretiens semi-directifs, menés en français, d'une durée médiane d'une heure et demie, ont été répétés et assortis d'observations dans un cadre familial dans un tiers des cas. Les récits de vie avaient pour objectif de retracer les parcours biographiques, de saisir les itinéraires migratoires et d'appréhender les imbrications entre les trajectoires individuelles et les histoires familiales. Ce matériau sociologique constitue la base d'une comparaison multidimensionnelle entre les parcours de vie de personnes ayant émigré à des périodes différentes (1951 à 2015), ayant vécu plus ou moins longuement à l'étranger (1 à 60 ans), en France principalement, mais pas exclusivement, et possédant des propriétés sociales contrastées (sexe, âge, position dans la famille, niveau de diplôme, profession, milieu social d'origine). Si tous ces enquêtés ne projetaient pas d'étudier à l'étranger, notre propos se concentrera ici sur les 87 enquêtés qui se sont déplacés, ou ont cherché à le faire, dans le cadre de leurs études. Tous ces enquêtés n'ont pas connu ce nouveau dispositif de contrôle (Campus France) et ils ont émigré dans des contextes légaux et locaux variables.

19. EduFrance, «Les étudiants étrangers en France : évolution et comparaison avec d'autres pays », Lettre d'EduFrance, 2006.

20. Satzewich (V.), «Visa Officers as Gatekeepers of a State’s Borders: The Social Determinants of Discretion in Spousal Sponsorship Cases in Canada », Journal of Ethnic and Migration Studies, 40 (9), 2014.

21. Mau (S.) et al., "New Control and Selectivity Arrangements ", in Mau (S.) et al., Liberal States and the Freedom of Movement, Basingstoke, Palgrave McMillan, 2012, p. 194.

22. Carling (J.), « Migration in the age of involuntary immobility: theoretical reflections and Cape Verdean experiences ", Journal of Ethnic and Migration Studies, 28 (1), 2002. 
L'intérêt d'un renversement de perspective, et d'une enquête auprès des demandeurs de visas, révèle ici toute sa pertinence. En analysant les perceptions et les pratiques de citoyens africains qui font avec cette politique du visa, et souvent ne parviennent pas à franchir la frontière légale, il devient possible de comprendre comment l'établissement de ces dispositifs locaux transforme inégalement les expériences de l'émigration étudiante. La comparaison multidimensionnelle, ici entre deux pays de départ et entre des enquêtés aux propriétés sociales contrastées, permet également de mieux saisir les distinctions qui s'opèrent à l'intérieur de cette population. La vision d'un obstacle indistinct, uniformément opposé partout et à tous dans les pays africains, peut ainsi être largement déconstruite. Le travail consulaire apparaît à la fois localement et socialement différencié. Selon leur pays d'origine, leurs propriétés sociales et leur histoire familiale, les étudiants parviennent inégalement à s'approprier la frontière, en mobilisant les ressources individuelles et collectives nécessaires pour se conformer à ces mécanismes de contrôle (I). L'examen de l'éventail des stratégies de conformations, d'accommodements ou de contournements ${ }^{23}$ de la procédure du visa démontre l'hétérogénéité de la catégorie des étudiants africains qui ne constitue pas un groupe monolithique. Face aux refus d'une demande de visa, les enquêtés démontrent une inégale aptitude à réorienter leur trajectoire migratoire (II). Dans le temps long des histoires individuelles, mobilités et immobilités spatiales deviennent des catégories poreuses. Dans cet article, nous ferons donc l'hypothèse que, malgré l'appartenance à la catégorie des «étudiants internationaux » soumis à des politiques a priori plus favorables, les étudiants africains n'en demeurent pas moins des étrangers comme les autres. Les opportunités d'internationalisation de leur parcours se jouent au gré des imbrications des frontières politiques et des frontières sociales.

\section{Mobiliser des ressources (im)matérielles face au consulat}

Après avoir obtenu une admission dans une université française, les étudiants s'engagent dans une procédure de demande de visa spécifique, «censée être plus protectrice ${ }^{24} »$. Mais les démarches qu'ils affrontent les soumettent in fine aux mêmes exigences sélectives que les autres candidats à l'émigration. Du fait de la diversité des ressources nécessaires au succès d'une demande, l'accès à la mobilité étudiante internationale se révèle socialement très sélectif. Ces disparités sont d'autant plus fortes que, depuis la mise en ouvre des politiques d'ajustements structurels en Afrique dans les années 1980, les États de départ n'octroient plus de bourses aux étudiants. Ces derniers doivent ainsi faire avec leurs ressources propres, ce qui tend à renforcer la logique «élitaire » de leurs mobilités ${ }^{25}$. Pour autant, les milieux populaires, qui rassemblent des "groupes

\footnotetext{
23. Barrault (L.), Gouverner par accommodements. Stratégies autour de la carte scolaire, Paris, Dalloz, 2013.

24. Belaïsch (S.), Petersell (L.), dir., Visa refusé..., op. cit., p. 6.

25. Hafaiedh (A.), Le visa étudiant. Objet de désir, miroir des « indésirables », Tunis, CERES, 2005, p. 95-112.
} 
sociaux caractérisés par une position matériellement et culturellement dominée dans l'espace social ${ }^{26}$, ne sont pas mécaniquement exclus de la mobilité étudiante.

\section{Affronter les coûts du visa}

La préparation du dossier de demande de visa étudiant est composée d'une série d'étapes, qui constituent autant de dépenses successives. La partie émergée du coût final réside dans les frais de dossier : 60 euros pour un visa court séjour et 99 euros pour un visa long séjour. Mais à ces frais directs s'ajoute un ensemble d'autres démarches à effectuer pour satisfaire aux exigences consulaires. Cette partie immergée demeure la plus coûteuse en temps et en argent. Les étudiants doivent souscrire, auprès d'agences agréées par le consulat, une assurance voyage pour leur rapatriement éventuel (30-60 euros) et fournir aux agents les justificatifs d'une réservation d'un vol aller-retour (900 à 2000 euros, selon le pays). L'attestation d'un hébergement pendant toute la durée du séjour est une autre pièce majeure. L'étudiant peut fournir la preuve d'une réservation hôtelière, d'une durée maximale de trois mois, parfois facturée par l'établissement. Il peut obtenir une attestation d'accueil par un particulier, délivrée par la mairie de la ville d'accueil (45 euros). Il peut encore tenter d'obtenir un logement en résidence universitaire, ce qui, du fait de la complexité des démarches à distance, laisse peu de chances aux étudiants non boursiers et non européens, qui ne sont pas prioritaires. Le fait de pouvoir justifier de «moyens d'existence » suffisants constitue le dernier élément central. Les agents consulaires attendent du demandeur qu'il puisse justifier d'un apport personnel à hauteur de 615 euros mensuels, ou du soutien d'un proche qui devra justifier ses liens de parenté. Cet appui doit être attesté par la présentation de relevés bancaires, qui garantissent que la somme totale est bloquée sur un compte (7 380 euros) ou établissent la preuve de la planification d'un virement bancaire mensuel. Le taux de bancarisation dans les deux pays ne dépassant pas $20 \%$, ces exigences financières obligent dans bien des cas les individus à ouvrir et à approvisionner un compte bancaire. En cas de refus de la demande, parfois annoncé tardivement, l'annulation des offres souscrites peut entraîner le paiement de pénalités, voire le non-remboursement des sommes engagées.

Les observations menées auprès d'étudiants issus des catégories populaires rurales en train de déposer leur demande de visa ont révélé que la constitution même du dossier a un coût non négligeable. Avant même de solliciter Campus France, il faut obtenir un acte de naissance, un certificat de nationalité, une

\footnotetext{
26. Schultheis (F.) et al., Les classes populaires aujourd'hui. Portraits de familles, cadres sociologiques, Paris, L'Harmattan, 2009, p. 14. Trois éléments caractérisent ces catégories populaires : " petitesse du statut professionnel ou social, étroitesse des ressources économiques - sans que cela signifie nécessairement précarité -, éloignement par rapport au capital culturel ». Schwartz (O.), «Peut-on parler des classes populaires ?», La Vie des idées, 2011.
} 
carte d'identité (10 euros) et un passeport (45-60 euros). Au-delà des "problèmes d'état civil » qui peuvent ralentir l'obtention de ces documents ${ }^{27}$, il est fondamental d'avoir accès à une connexion Internet pour se documenter et télécharger les différents dossiers d'inscription, de faire des photographies d'identité conformes chez un photographe, de se rendre dans un cybercafé équipé d'un photocopieur pour dupliquer autant de fois que nécessaire toutes les pièces justificatives, etc. Pris indépendamment, ces frais annexes n'excèdent guère quelques euros, mais peuvent devenir rédhibitoires pour des étudiants qui habitent loin de la capitale et doivent multiplier les allers-retours en transports collectifs entre la banque, les différentes administrations et l'espace Campus France.

Mis bout à bout, les coûts financiers auxquels les personnes font face pour pouvoir étudier à l'étranger s'élèvent dans le meilleur des cas à 3000 euros pour un visa court séjour, et à près de 10000 euros pour un visa long séjour. L'ampleur de ces coûts apparaît d'autant plus considérable si l'on garde à l'esprit que, d'après les données de la Banque mondiale, en 2014, le salaire mensuel moyen était de 70 euros aux Comores et de 42 euros au Togo.

Chez les étudiants dont les parents font partie de la bourgeoisie africaine ${ }^{28}$, qui cumulent le prestige des emplois occupés, l'importance des revenus économiques et du patrimoine accumulé (commerçants internationaux, cadres d'entreprises privées ou de sociétés publiques, chefs d'entreprise ou professions libérales), l'acquisition d'un visa peut être envisagée sereinement. Rares sont les enquêtés issus des milieux favorisés qui, à l'instar de Radia N., fille d'un diplomate et d'une magistrate, ont livré les détails financiers de leur départ. En 2001, après s'être inscrite en licence d'AES à Tours, Radia a rempli son dossier de visa avec sa mère, qui avait elle-même étudié en France, et s'est rendue au consulat une seule fois pour fournir toutes les pièces justificatives. Son billet d'avion avait été acheté par cette dernière, qui avait par ailleurs ouvert en France un compte bancaire et un compte épargne bloqué au nom de sa fille, avec de l'argent disponible pour ses trois premiers mois : «Disons que j’avais des fonds », résumet-elle (Radia N., 29 ans, émigrée togolaise, DEA de commerce, employée d'une entreprise de services commerciaux en ligne).

Cela ne signifie pas pour autant que les individus dont les revenus sont bien moindres et qui ne disposent pas d'épargne soient complètement mis à l'écart de ces démarches financièrement exigeantes. Mais qu'il s'agisse de familles populaires ou de familles aux positions sociales médianes dont les emplois plus prestigieux demeurent irrégulièrement rémunérés (employés des secteurs

27. Goldberg (D.) et al., Rapport d'information du Groupe d'amitié France. Union des Comores présenté à la suite de la mission effectuée du 2 au 11 octobre 2010, Paris, Assemblée nationale, 2010.

28. Lesourd (M.), "Mondialisation et nouvelle mobilité des élites africaines. La mobilité bourgeoise au Sud », ÉchoGéo, 20, 2012. 
publics et privés, enseignants, journalistes ou artistes), la mobilisation des ressources économiques emprunte des temporalités plus longues. Dans ce cas, les étudiants sont contraints d'avoir recours à des soutiens financiers ponctuels, soit dans un cercle familial élargi, soit par le biais d'épargnes collectives, comme l'ont fait Nakib B. et son frère aîné. Leur mère a quitté son poste de secrétaire dans la fonction publique et demandé à percevoir tous ses arriérés de salaires, soit plus de 1500 euros. Avec cette somme, elle a scolarisé Nakib dans une école publique et son frère aîné dans un collège privé. Quand l'aîné a obtenu son baccalauréat, leur père, ancien agriculteur devenu musicien, a organisé une cotisation auprès des membres de son orchestre. Grâce à cette contribution qui a permis de réunir plus de 1100 euros, le départ du fils aîné, parti étudier l'agronomie à Paris, a pu être en partie financé. Mais cette somme était insuffisante pour aider à son tour Nakib, qui a dès lors arrêté sa scolarité et entrepris une formation en plomberie à Madagascar (Nakib B., 30 ans, émigré de retour comorien, formation en installation sanitaire, musicien).

Quels que soient les milieux sociaux enquêtés, nombre de familles investissent symboliquement et matériellement des stratégies migratoires éducatives pour leurs enfants, mais la possession d'un capital économique conséquent continue d'être le premier obstacle à un départ effectif.

\section{Bénéficier d'un capital social et migratoire, ici et là-bas}

Avant d'engager une demande, il est préférable de s'assurer de soutiens ponctuels. Les relations sociales sur lesquelles peuvent s'appuyer les étudiants sont d'autant plus déterminantes qu'elles s'étendent à la fois dans le pays d'origine et dans le futur pays d'accueil. Mais ce possible appui sur un capital social international est inégalement réparti dans l'espace social.

Dans les milieux sociaux aisés, la pratique de la mobilité est fréquente. Les parents ont eux-mêmes souvent été des étudiants internationaux avant de prendre en charge les démarches de leurs enfants. Il est donc plus courant de disposer de contacts solides auprès de citoyens français, qui sont mis à contribution au moment du départ. Ainsi, Kossi K. a pu bénéficier des relations de ses parents, deux avocats togolais, qui ont étudié à Poitiers et avaient gardé nombre d'amis poitevins travaillant eux-mêmes dans le domaine juridique. Ces derniers ont pris une part active dans l'émigration de Kossi, en l'encourageant à étudier à l'étranger, en l'aidant au moment de s'inscrire en faculté de droit, en certifiant d'un hébergement conforme aux attentes consulaires et en l'aiguillant après son arrivée en 1992. Les ressources financières parentales, augmentées de solides appuis amicaux en France, ont donc assuré la réussite de sa demande de visa (Kossi K., 36 ans, émigré de retour togolais, non-diplômé en droit, musicien).

Hors de ces milieux élitaires, plus rares sont les familles qui peuvent compter sur de tels soutiens à l'étranger. Pourtant, même dans les familles populaires peu mobiles, quelques jeunes ayant participé à des activités associatives et culturelles 
ont réussi à nouer des liens amicaux ou sentimentaux avec des citoyens français, qui se sont avérés cruciaux au moment des préparatifs de leur émigration. Au début des années 1990, Mdjahazi M. était un jeune lycéen. Fils d'une couturière qui dirigeait une école coranique et d'un agriculteur, Mdjahazi participait à des travaux de maçonnerie dans sa ville, pour gagner un peu d'argent. C'est sur l'un de ces chantiers qu'il a rencontré un groupe de jeunes ingénieurs français venus construire une école et s'est lié d'amitié avec l'un d'eux. Le père de Mdjahazi gagnait 3 à 6000 euros par an grâce à la vente de vanille et a pu lui payer son billet d'avion pour venir en France. Mais c'est cet ami français qui lui a trouvé une école toulousaine l'autorisant à intégrer une formation agricole, sans avoir préalablement passé son baccalauréat, et lui a assuré un hébergement dans sa famille à Montpellier (Mdjahazi M., 40 ans, émigré comorien, baccalauréat agricole, maçon).

Mais ce qui permet l'émigration d'étudiants disposant de faibles ressources économiques, c'est l'existence préalable d'une dispersion transnationale de la famille $^{29}$, qui s'avère plus fréquente aux Comores où la décolonisation tardive (1975) a plus longtemps facilité les départs d'émigrés plus précaires. Dans ces familles populaires rurales qui comptent un émigré pionnier parti grâce aux opportunités professionnelles du tournant de la décolonisation (embauches dans l'armée ou les compagnies maritimes), ou grâce à la vente d'une partie du petit patrimoine foncier possédé, ce capital migratoire ancien est déterminant. Quand un ou plusieurs parents, aînés ou proches vivent déjà à l'étranger, il est possible de négocier avec eux un soutien matériel et financier, qui peut compenser l'absence de ressources dans l'espace local. Dans ce cas, les projets migratoires se construisent souvent en combinant l'aide d'un proche dans le pays d'origine et d'un proche émigré en France. C'est ce qu'a vécu Nadjati A., fille d'agriculteurs, en partant étudier à Montpellier en 2006, après son baccalauréat. Elle a pu émigrer grâce à l'appui financier d'un oncle commerçant aux Comores, qui s'est porté garant à la place de ses parents. Son frère, ancien étudiant devenu employé de la mairie montpelliéraine, qui avait lui-même pu émigrer grâce au soutien d'une tante vivant à Marseille, appuie les démarches de Nadjati, en louant un appartement à son nom. En retour, Nadjati promet qu'elle aidera leur benjamine, après son baccalauréat. Elle reconnaît cependant que «malheureusement, c'est pas tout le monde qui a quelqu'un là-bas, qui puisse se déplacer, regarder, chercher les inscriptions, se porter garant pour l'appartement, c'est pas tout le monde qui a cette chance. Parce qu'il faut des sous. Faut que y'ait des gens qui puissent t'aider, ça c'est sûr et certain. Et ceux qui n'ont personne n’ont pas trop de chances... » (Nadjati A., 24 ans, émigrée comorienne, bachelière, étudiante en ressources humaines). Si les étudiants des familles populaires dispersées attendent une mobilisation de leurs proches

29. Touré (N.), «L'approche “réseaux” dans les études migratoires », e-Migrinter, 13, 2015. 
en France, les observations menées au sein des familles dans le pays d'origine soulignent que ce soutien n'a toutefois rien de mécanique et peut susciter des conflits dans l'entourage.

Les conditions imposées au garant de l'hébergement, qui doit lui-même fournir des documents relatifs à son identité, à ses revenus et à la nature de son logement, sont nombreuses. Connaître un citoyen français ou un immigré en France n'est donc pas un gage de succès en soi. Les agents consulaires examinent les garanties financières qu'il peut offrir, pour vérifier qu'il satisfait bien aux conditions d'un hébergement convenable. D’après le consul-adjoint de France à Moroni, il s'agit d'éviter des situations dans lesquelles l'émigré se retrouve dans « un logement tout petit, à six ou sept dedans", parce que «le tonton, le frère, le cousin et autres accueillent ». C'est sur ce point que des inégalités persistent dans l'accumulation d'un capital migratoire ou d'un capital social international. Les classes supérieures restent ici favorisées dans ces démarches, parce qu'elles disposent de la «force des liens forts ${ }^{30}$ ", quand les autres demandes peuvent être écartées du fait des représentations négatives associées à ces pratiques d'entraide.

\section{Maîtriser la langue française}

Les agents consulaires attendent des étudiants désireux de poursuivre leur cursus en France qu'ils maîtrisent le mieux possible la langue française. Cette exigence, qui par certains aspects peut paraître incongrue ${ }^{31}$, vise à s'assurer que les étudiants pourront suivre les enseignements dispensés, et plus généralement que les difficultés linguistiques ne constitueront pas un obstacle à leur intégration, le temps du séjour. Contrairement aux autres candidats à l'émigration, la sélection des dossiers des étudiants s'effectue donc explicitement au regard de la possession de ressources tout à la fois matérielles et immatérielles. Au cours des entretiens individualisés dans l'espace Campus France, les étudiants doivent ainsi s'exprimer «correctement». En racontant son départ, Nadjati A. entremêle les obstacles culturels (la langue), scolaires (le cursus) et économiques (l'hébergement) qui ont pu motiver son premier refus de visa, mais aussi ceux qu'ont affrontés certains de ses amis : "C’est tendu pour ceux qui ne maitrisent pas vraiment la langue. Moi, je maitrise pas très bien, mais au moins je parlais quand même [rires]. J'avais pas peur de parler en français. Y'en avait d'autres qui étaient beaucoup plus tendus, même mes amis. D'autres qui avaient des hébergements et tout et tout, c'était beaucoup plus compliqué parce que y'avait beaucoup de conditions à respecter et... malheureusement c'était pas du tout

\footnotetext{
30. Wagner (A.-C.), Les classes sociales dans la mondialisation, Paris, La Découverte, 2007, p. 49.

31. Cette injonction paradoxale est résumée par la phrase de l'humoriste Coluche, citée par Serge Slama : «Étudiants immigrés en France, attention, désormais pour apprendre le français en France, il faudra savoir le français!»
} 
ça, donc ils étaient refusés. Le visa était vraiment refusé » (Nadjati A., 24 ans, émigrée comorienne, bachelière, étudiante en ressources humaines).

Attente tacite des agents, la maîtrise du français introduit une nouvelle inégalité entre les demandeurs. La scolarisation dans des établissements privés, au sein desquels les enseignements sont dispensés par des enseignants français ou ayant eux-mêmes émigré, et dans lesquels les élèves fréquentent des élèves nationaux, binationaux ou étrangers très mobiles est un atout. Autrefois réservées aux fractions des catégories supérieures les mieux dotées en ressources économiques, ces scolarités aussi prestigieuses qu’onéreuses sont désormais investies par des familles aux positions sociales plus modestes, quand les membres émigrés prennent en charge à distance la scolarité de leurs enfants ou cadets. Toutefois, l'enquête a montré que les familles élitaires restent les seules à renforcer les socialisations linguistiques acquises au cours de ces apprentissages scolaires par une fréquentation active des centres culturels français (bibliothèque, cours du soir, spectacles, etc.) et une familiarisation avec l'actualité française internationale par le biais des chaînes d'informations en continu. Dans cet horizon quotidien très internationalisé, la pratique du français va de soi et se trouve renforcée par des séjours réguliers à l'étranger.

Dans les familles qui ne disposent ni de fortes ressources économiques ni d'un capital migratoire, quand les enfants sont engagés dans un processus de scolarisation, ils le sont majoritairement dans des établissements publics. L'ensemble des enseignements n'y est pas toujours assuré en français et les sociabilités scolaires limitent les occasions de parler français en dehors de l'école. Ces étudiants peuvent à terme se sentir moins compétents, et dès lors moins aptes à faire preuve d'une aisance orale en français. Pour compenser ce stigmate face aux agents, ils peuvent prendre des cours et obtenir une certification officielle, par des diplômes élémentaires de langue française (DELF). Mais il s'agit là d'un investissement coûteux qui nécessite de payer plusieurs centaines d'euros pour s'inscrire, suivre des cours et passer un examen final à l'Alliance Française de Moroni ou à l'Institut français de Lomé.

À cet égard, le profil même des émigrations étudiantes aux Comores et au Togo produit des suspicions contrastées. Si la grande majorité de ces étudiants s'inscrit à l'université ( 80 et $86 \%$ ), tous ne partent pas au même moment de leur cycle d'études. L'université de Lomé étant ancienne (1970) et relativement développée, près de la moitié des étudiants togolais intègre directement un master en France. L'université des Comores étant récente (2003), deux tiers des étudiants comoriens partent après le baccalauréat et souhaitent intégrer une licence, et sont donc dotés d'un niveau de français plus faible à faire valoir face aux agents ${ }^{32}$.

32. D'après le PNUD, le niveau d'instruction des migrants comoriens correspond aux autres pays maghrébins et subsahariens, là où le Togo fait partie des pays africains dans lesquels les émigrés sont les plus scolarisés et diplômés : niveau primaire ( $28 \%$ au Togo, $64 \%$ aux Comores), niveau secondaire (34 \% au Togo, 


\section{Ne pas éveiller les soupçons}

L’octroi d'un visa ne répond pas seulement à des critères codifiés et juridiquement définis. Comme l'ont montré les recherches sur les guichets consulaires, il se construit dans les interactions entre les agents chargés du contrôle et les demandeurs. Avant la généralisation des entretiens auprès de Campus France, l'individualisation des dossiers produisait déjà des rencontres, plus ou moins formelles, qui étaient autant de moments pendant lesquels les agents questionnaient les candidats sur leur profil familial, la cohérence de leur parcours scolaire, de leurs motivations personnelles et de leurs aspirations professionnelles. Dans les faits, comme les agents consulaires le font avec tout demandeur de visa, l'examen des dossiers est guidé par le contrôle du "risque migratoire ", c'est-àdire la crainte d'un "détournement de la procédure de visas de court séjour à des fins de maintien sur le territoire ${ }^{33} "$, jugé particulièrement sensible dans les pays africains, et plus encore aux Comores qu'au Togo. Tout peut alors prêter à suspicions : l'authenticité des documents présentés, la tangibilité des soutiens financiers attendus à l'étranger, le réalisme des objectifs professionnels, la sincérité des attaches familiales dans le pays d'origine et des intentions de retour.

En haut de l'échelle sociale, il n'est pas toujours nécessaire de chercher à rétablir « la confiance dans l'individu après l'épreuve du soupçon porté sur un ensemble national ${ }^{34}$ ", tant les étudiants et leur famille jouissent de ce lien de confiance dès leur présentation auprès des agents. Leur ancrage social, leurs ressources et leur ascendance les éloignent d'une vision misérabiliste et de suspicions sécuritaires. Radia N. décrit ainsi la facilité des démarches administratives au Togo pour se procurer sa carte d'identité et son passeport, puis obtenir son visa (29 ans, DEA de commerce, employée d'une entreprise de services commerciaux en ligne). Elle attribue cette bienveillance à la réputation de sa mère magistrate.

- Mais avant d'arriver à Tours quand même, il faut dire que bon, contrairement à beaucoup de Togolais, moi j'ai eu la chance, déjà à l'étape visa pour venir. Ça a pas été galère quoi, parce que faut qu'on se le dise obtenir un visa pour venir en France ça peut être un long parcours du combattant.

- T'as pas fait la queue devant le consulat...

- Voilà, bon j'ai la chance d'avoir une mère qui a un certain nom au Togo qui a fait que... voilà quoi... mes démarches... je me suis pointée moi, une fois... c'est triste à dire mais oui j'ai eu des passe-droits, voilà. J'ai eu des passe-droits

\footnotetext{
$26 \%$ aux Comores), études supérieures (36\% au Togo, $11 \%$ aux Comores). Klugman (J.), dir., Rapport mondial sur le développement humain. Lever les barrières: mobilité et développement humains, New York, PNUD, 2009.

33. Gouteyron (A.), Trouver une issue au casse-tête des visas, Paris, Commission des finances, Sénat, 1997, p. 18.

34. Bigo (D.), « Le visa Schengen et le recours à la biométrie », in Crettiez (X.), Piazza (P.), dir., Du papier à la biométrie. Identifier les individus, Paris, Presses de Sciences Po, 2006, p. 254.
} 
comme cela peut arriver... Ah je crache pas dessus hein, franchement, j'ai pas honte de le dire, j'ai eu cette chance, j'en ai profité. Donc non, j'ai pas connu les longues files d'attente devant le consulat... bon j'ai patienté un peu quand même, mais c'est tout quoi [rires]... j'arrivais, je posais le dossier, j'étais à la maison et j'avais les papiers qui arrivaient.

- Sans trop d'angoisse...

- Voilà, sans trop d'angoisse, je savais que ça irait.

«Lieu de passages négociés» pour certains ${ }^{35}$, les consulats et les espaces Campus France deviennent un lieu de défiances répétées pour les moins dotés. Le décalage entre la faiblesse des ressources économiques et culturelles et la volonté de poursuivre des études à l'étranger oblige les étudiants à adopter une présentation de soi qui n'éveille aucun soupçon, à produire des « histoires plausibles » au regard des attentes des agents ${ }^{36}$ et à démontrer leur " bonne foi ${ }^{37}$ ", pour ne pas devenir les figures incarnées - et genrées - de l'émigré qui chercherait à travailler ou de l'émigrée qui voudrait rejoindre son conjoint en France ${ }^{38}$, en se faisant passer pour des étudiants. Les entretiens peuvent être vécus par les demandeurs, à la fois comme un « examen de passage » et comme un moment où se construit, dans le rapport ordinaire à l'agent, une « expérience d'altérité $^{39}$ ", altérité équivoque, tout à la fois sociale, culturelle et symbolique.

Quand ils ont spontanément évoqué leur passage au consulat, les enquêtés issus de familles économiquement modestes, mais plus dotés en ressources scolaires et culturelles, ont tous souligné qu'ils se trouvaient soumis à des décisions qui leur paraissaient arbitraires, faute de justification ${ }^{40}$. Ils ont également évoqué les longues files d'attente, gérées aléatoirement par des gardiens "pas commodes » et les obligeant à venir dès cinq heures du matin, les relations « difficiles » et le mauvais accueil qui leur était réservé par des agents qui leur ont « mal parlé ». Ces critiques allusives ne disent sans doute pas toute la variété des relations entretenues au guichet. Faute d'une observation des interactions entre agents et étudiants, on peut simplement conclure que face à des démarches de plus en plus coûteuses et des exigences draconiennes, la fortune d'une demande de visa étudiant est à géométrie variable. Elle se joue dans la combinaison de

35. Darley (M.), « Passages négociés à la frontière Schengen », Plein Droit, 84, 2010, p. 8.

36. Heyman (J.), « Risque et confiance dans le contrôle des frontières américaines », Politix, 87 (3), p. 36.

37. Les instructions européennes données aux consulats des pays membres encouragent les agents à « exercer une vigilance particulière sur les "populations à risque", chômeurs, personnes démunies de ressources stables, etc. En cas de doute [...] la représentation diplomatique ou consulaire s'abstiendra de délivrer le visa. À l'inverse, les contrôles seront allégés pour les demandeurs reconnus comme étant des personnes bona fide». Guiraudon (V.), « Une obsession : la clandestinité », Plein droit, 57 (2), 2003.

38. Infantino (F.), «Gouverner les frontières ou appliquer des droits ? Le contrôle des mariages aux consulats de Belgique, d'Italie et de France à Casablanca », Migrations Société, 6 (150), 2013.

39. Hafaiedh (A.), Le visa étudiant. Objet de désir, miroir des «indésirables », op. cit., p. 172.

40. "Il ressort que le hasard, l'arbitraire et le jeu des influences parasitent le processus au détriment des étudiants ». Cerisier-Ben Guiga (M.), Blanc (J.), L'accueil des étudiants étrangers en France, Rapport d'information, Sénat, 2005. 
multiples ressources (économiques, sociales, migratoires, scolaires, culturelles et symboliques), ce qui place les individus issus des milieux populaires en première ligne des demandeurs suspectés, même lorsqu'ils disposent de soutiens familiaux à l'étranger et qu'ils ont été plus longuement scolarisés.

L'épreuve du visa étudiant semble finalement équivoque au sein même des étudiants africains. En effet, les représentations diffuses des acteurs français chargés de gérer ces deux migrations - comoriennes et togolaises - demeurent largement opposées. Là où l'invisibilité sociale et l'intégration des migrants togolais sont souvent vantées, c'est l'altérité des migrants comoriens qui est mise en avant. Ainsi, les agents interrogés dressent le portrait d'une migration togolaise composée de " gens d'un certain niveau ", qui forment « une communauté paisible, qui ne pose pas de problème particulier ${ }^{41} »$. En miroir, plusieurs arguments récurrents viennent dessiner les contours d'une migration comorienne dont la présence est décrite comme problématique : poids de l'islam, faible niveau social des migrants et caractère communautaire de leur installation en France. On peut dès lors faire l'hypothèse que ces soupçons différenciés, et cette plus ou moins grande indésirabilité des demandeurs, peuvent expliquer à la fois la convocation systématique en entretien des citoyens comoriens (là où seuls $80 \%$ des Togolais sont invités à se présenter au consulat), mais aussi les différences dans les taux d'acceptation des visas. Si la France est généralement présentée comme un pays dont les rejets de demandes de visas sont "globalement faibles ${ }^{42}$ ", les taux de refus varient considérablement d'un pays d'origine à l'autre. D'après les données obtenues auprès des consulats, sur la période 2004-2008, les taux de refus étaient compris entre $17 \%$ et $23 \%$ au Togo. En 2006, le consulat de France à Moroni se situait quant à lui à la $9^{e}$ place des consulats qui refusaient le plus de dossiers (31\%), ce taux s'élevant à $55 \%$ de 2009 à $2011^{43}$.

\section{Réorienter sa route migratoire}

La propension à la mobilité internationale des étudiants varie considérablement aux Comores et au Togo. Ainsi, en 2013-2014, 5,6 \% des étudiants togolais et $66,6 \%$ des étudiants comoriens partaient poursuivre leur cursus à l'étranger ${ }^{44}$. En revanche, la place de la France, ancienne puissance coloniale

\footnotetext{
41. Ces termes se retrouvent en partie dans un rapport parlementaire. Vinçon (S.), Rapport sur le projet de loi autorisant l'approbation de la convention entre le gouvernement de la République française et la République du Niger relative à la circulation et au séjour des personnes, Paris, Commission des Affaires étrangères, Sénat, 1997.

42. Gouteyron (A.), Trouver une issue au casse-tête des visas, op. cit., p. 17.

43. Si l'espace Campus France de Lomé n’a pas communiqué les données relatives aux demandes des étudiants togolais, les données obtenues à Moroni soulignent que ces ordres de grandeur se retrouvent dans le cas du visa étudiant, le taux de refus étant de $60 \%$ des demandes en 2008.

44. Soit 3710 sur 66500 étudiants au Togo, et 4151 sur 6231 étudiants aux Comores, où l'unique université a été créée en 2003.
} 
dans les deux cas, demeure centrale dans cette géographie internationale. Cette destination est choisie par $27 \%$ des étudiants togolais et $30 \%$ des étudiants comoriens et son attractivité n'a cessé d'augmenter entre 2010 et $2015^{45}$. Les diplômes qui y sont délivrés sont présentés au fil des entretiens comme "les plus considérés ", associés à "un label de qualité ». Dès lors, quelles sont les possibilités qui s'offrent aux étudiants lorsqu'ils se heurtent à la frontière et que leur visa est refusé ? La seule issue réside-t-elle dans l'alternative entre un renoncement au départ et un recours légal dont les enquêtés anticipent généralement l'échec? Au regard des parcours des étudiants et de leur famille, il apparait que les déplacements locaux, nationaux, régionaux et internationaux s'imbriquent dans des parcours peu linéaires, et complexifiés par cet affermissement continu des contrôles consulaires.

\section{Entrer dans l'illégalité}

La première hypothèse consiste spontanément à envisager que les demandeurs refoulés se tournent vers un départ illégal. Nombre d'étudiants interrogés mettent pourtant nettement à distance ces pratiques migratoires illicites, d'autant plus lorsqu'ils font partie de familles socialement aisées. D’autres, plus familiarisés avec ces contournements des procédures légales, s'avèrent moins réticents, comme le révèle le cas de Bakri S. et le récit qu'il fait du départ de ses deux sœurs aînées, qui ont émigré grâce à l'achat de faux papiers. Alors que son frère s'est récemment vu refuser un visa court séjour pour rendre visite à leurs sœurs, Bakri, seul bachelier de la famille et récent diplômé, souhaite quant à lui faire valoir sa réussite scolaire, pour poursuivre son cursus en France (Bakri S., 23 ans, non-émigré comorien, licence de géographie, sans-emploi). Mais s'il écarte dans un premier temps cette option illégale, c'est avant tout parce que l'entrée dans la clandestinité est elle aussi très sélective. Pour acheter de faux papiers d'identité, obtenir le droit d'utiliser les papiers d'un autre, organiser un faux mariage ou corrompre un agent consulaire, il faut disposer de ressources financières très importantes. La mère de Bakri, petite commerçante, a ainsi payé plus de 7000 euros pour acheter de faux papiers à ses filles. Les prix évoqués peuvent dans certains cas aller bien au-delà de ces sommes (10 à 20000 euros) et dépasser largement les montants nécessaires à un départ légal. De telles pratiques supposent en outre des ressources relationnelles pour pouvoir être mis en contact avec des individus qui organisent ces trafics de papiers. Un certain savoir-faire est également indispensable pour distinguer les personnes fiables de celles qui réclament de l'argent sans réelle contrepartie. La faible maitrise de ces savoirs peut être financièrement coûteuse, comme l'a expérimenté la famille de Bakri lorsqu'elle a voulu organiser le premier départ de la fille aînée. En 1998, cette dernière a pris contact avec un habitant d'une ville voisine qui « envoyait

45. Le nombre d'étudiants inscrits en France est en forte augmentation au Togo $(+31,5 \%)$, comme aux Comores (+45\%). 
les gens sans visa ». Leur mère lui a donné 3000 euros, économisés grâce à la vente de poissons sur le marché. "Seulement, elle savait pas les démarches, le mec a pris l'argent, il a pris la fuite ", raconte Bakri. Après deux ou trois jours d'attente, leur mère a envoyé un ami menacer l'auteur de cette escroquerie. À défaut de l'organisation du départ en France de sa fille, elle a obtenu 1000 euros, une voiture et un bœuf en guise de dédommagement.

\section{Renoncer au départ}

Refusant des départs irréguliers qu'ils seraient bien incapables de mettre en œuvre, beaucoup d'étudiants d'origines populaires sont captifs des démarches liées au visa étudiant. En cas de refus, ils n'ont pas toujours d'autres options que de renoncer au départ, ou en tout cas de le différer d'une ou plusieurs années ${ }^{46}$. Mais les trajectoires sociales liées aux renoncements à la mobilité spatiale sont extrêmement hétérogènes d'une famille à l'autre.

Certains renoncent parce qu'ils n'ont pas d'appui suffisant à l'étranger pour les aider à s'organiser, mais obtiennent en revanche des soutiens sur place. C'est le cas de Marcel N., dont les trois frères et sœurs émigrés n'ont pour le moment rien fait pour l'aider à partir à son tour. Parallèlement à ses études, ce dernier travaille comme comptable dans l'entreprise de conditionnement d'eau en sachets dirigée par son frère aîné, le seul de la fratrie, avec Marcel, à ne jamais avoir quitté le Togo. Marcel est payé trois fois plus que le SMIG (90 000 francs CFA, 137 euros), ce qu'il juge "plutôt bien ». Son frère s'étonne d'ailleurs de voir Marcel vouloir partir et lui répète régulièrement qu'ils ont « le potentiel ici pour tout faire » (Marcel N., 24 ans, non-émigré togolais, étudiant en biologie).

D'autres different leur départ dans la mesure où leur diplôme leur permet, pour un temps, d'éviter de vivre les mêmes situations d'inactivité ou de sous-emploi qu'expérimentent leurs proches peu scolarisés. Après avoir vu sa demande de visa refusée pendant l'été, Bakri S. a obtenu un poste d'enseignant dans un collège public de sa ville. Soulagé dans un premier temps, il sait toutefois que l'irrégularité de ses revenus ne pourra pas être une situation acceptable à terme, d'autant moins que sa femme et lui ont eu une fille, et il n'a donc pas pleinement renoncé à son projet d'étudier à l'étranger, où il espère pouvoir travailler en parallèle de sa formation.

Mais à ces cas dans lesquels les projets étudiants ont laissé place à des projets professionnels plus ou moins satisfaisants s'opposent une majorité d'enquêtés issus de familles populaires, souvent rurales, qui ne trouvent aucun emploi

46. Campus France encourage progressivement les étudiants à effectuer d'abord une licence dans leur pays d'origine, et les incite à candidater à partir d'un master. Ainsi, aux Comores, en 2011-2012, $81 \%$ des entretiens avec des étudiants de second cycle ont mené à un dépôt de dossier, contre $22 \%$ pour les étudiants candidats à un premier cycle. 
et vivent cette non-reconnaissance de leur diplôme (baccalauréat ou licence) comme un déclassement social, sans pouvoir pour autant envisager une émigration étudiante.

\section{S'accommoder du visa}

En anticipant les causes d'un potentiel refus ou en réajustant à la marge leurs demandes, certains étudiants cherchent à s'accommoder de l'obstacle que représente pour eux le visa. Qu'il s'agisse de leur première demande ou de démarches renouvelées, ces enquêtés ont fait part de leurs stratégies pour mettre toutes les chances de leur côté, « tactiques » qui ne sont pas accessibles aux autres demandeurs de visa ${ }^{47}$. Beaucoup d'étudiants abandonnent leurs ambitions initiales, pour choisir des disciplines qu'ils estiment plus valorisées par les agents consulaires au moment de l'examen des dossiers. Essenam A., ancienne apprentie coiffeuse devenue étudiante en France, raconte que sa sœur cadette voulait étudier la médecine (Essenam A., 26 ans, émigrée de retour togolaise, doctorante). Plusieurs de ses amis l'ayant encouragée à déposer des demandes d'inscription dans un cursus d'AES qui leur semblait plus propice à l'obtention d'un visa, celle-ci a réorienté son projet étudiant. Élevée dans une famille de petits agriculteurs et ne disposant plus du soutien d'Essenam revenue vivre au Togo avec son mari français, son profil populaire ne lui a finalement pas permis d'obtenir un visa. Ces stratégies de réajustement des ambitions disciplinaires valent particulièrement pour les étudiants comoriens qui s'inscrivent davantage dans des diplômes dépréciés (31 \% en lettres, langues et sciences sociales) tandis que les étudiants togolais optent plus souvent pour des cursus valorisés ( $24 \%$ en économie, gestion et administration).

Outre les disciplines, les étudiants ciblent également des villes et des universités qui leur paraissent plus opportunes, au gré de leurs échanges avec leurs camarades étudiants. Récent diplômé de l'université des Comores et issu d'une famille d'agriculteurs, Seydalo I. raconte qu'il aimerait beaucoup étudier à Montpellier 3 ou à Paris 1. Mais il a entendu dire que ces deux universités étaient particulièrement sélectives pour les étudiants étrangers : «Là-bas, nul n’entre sauf des gens comme toi. » Pour plus de sûreté, il préfère donc déposer des dossiers d'inscription dans d'autres universités (Lyon, Mulhouse ou Annecy), qu'il pense moins exigeantes dans leur examen des dossiers. Plus qu'une véritable connaissance des critères de sélection universitaire, ce choix de villes révèle une intériorisation des récits d'émigrés entendus dans sa ville d'origine, parmi lesquels une majorité vit à Lyon et Annecy. Cette faible maitrise des savoir-faire migratoires s'observe à nouveau lorsque Seydalo m'amène un peu à l'écart de ses amis pour me demander, sur un ton inquiet, si la mauvaise note obtenue en

47. Couëdel (R.), "Savoir émigrer : projet d'études et projet migratoire des étudiants kabyles », L'Année du Maghreb, 3, 2007. 
géographie française lors de sa première année de licence pourrait être préjudiciable et constituer un critère de refus de sa demande de visa (Seydalo I., 24 ans, non-émigré comorien, licence de géographie, sans-emploi).

Ces différentes options aux issues très aléatoires dans les familles les moins dotées sont au contraire plus souvent couronnées de succès dans les familles aisées. En 2000, après un baccalauréat littéraire obtenu dans un prestigieux lycée privé des Comores, Amir S. s'est inscrit dans un DEUG d'histoire à l'université de Créteil. Ce cursus était un choix par défaut, puisqu'il a lui aussi suivi les conseils d'amis qui lui ont suggéré de choisir une discipline peu demandée et dans laquelle il avait de bons résultats scolaires: «Visiblement c'est pas une filière où je risquais d'avoir des refus d'admission » (Amir S., 30 ans, émigré de retour comorien, master professionnel en communication, chef d'entreprise). On notera que, dans son cas, c'est l'absence d'admission par une université française qui est redoutée, et non un refus consulaire. Peu de temps après son arrivée en France, Amir a abandonné ce cursus qui ne l'intéressait plus, pour un BTS en communication des entreprises. Plus encore que cette stratégie disciplinaire, c'est le soutien de son père, cadre de la fonction publique ayant exercé des fonctions politiques avant de devenir dirigeant de plusieurs structures privées, qui semble avoir été déterminant dans le succès de sa démarche auprès du consulat.

\section{Émigrer par étapes}

Face aux refus - réels ou anticipés - du consulat, les étudiants peuvent également envisager d'émigrer par étapes, c'est-à-dire de partir d'abord dans un autre pays africain, considéré comme une destination moins légitime (Madagascar, Égypte ou Sénégal aux Comores ${ }^{48}$; Maroc et Sénégal au Togo), pour réussir à terme à venir en France. Cette stratégie a été observée principalement dans les milieux populaires comoriens et n'est concevable que dans des familles déjà fortement dispersées. Mais le succès d'une telle entreprise dépend à la fois des ressources des proches et de leur volonté effective de participer à ce projet migratoire. Quand les parents ne peuvent pas ou ne cherchent pas à apporter cette aide, les étudiants se trouvent obligés de rester vivre dans le lieu qui était perçu comme une étape, ou de rentrer dans leur pays d'origine. Naïm H., benjamin d'une famille rurale comorienne a vécu cette contrainte au cours de son parcours. Trois de ses aînés ont émigré en France. L'aîné a poursuivi ses études jusqu'au doctorat ; le cadet, passé par Madagascar, a été employé par plusieurs restaurants avant de travailler comme ouvrier du bâtiment; le troisième est devenu ingénieur. À distance, tous les trois ont matériellement soutenu la scolarité de Naïm, dans des écoles publiques, puis dans un lycée privé pour parfaire sa maîtrise du français. Après son baccalauréat, obtenu après sa

48. Dans le cas comorien, Mayotte - île de l'archipel des Comores restée française en 1975 - fait partie intégrante de ces destinations délégitimées et perçues comme des étapes provisoires. 
deuxième année de terminale, ses frères lui promettent de l'inscrire dans une université française. Convaincu que ses frères "n'ont pas fait les choses comme il fallait », Naïm n'a obtenu aucune admission. Il passe une année blanche, sans inscription ni emploi. Alors qu'il souhaite rejoindre ses frères en France, ces derniers envisagent d'abord un départ moins coûteux. Sur les conseils d'une jeune émigrée de retour de son village, qui a étudié à Dakar et considère que les études y « sont sérieuses ", Naïm suggère le choix du Sénégal à ses frères et s'inscrit dans une formation en commerce, plus courte, afin de ne pas dépendre trop longtemps de sa fratrie. Naïm s'imagine obtenir son diplôme, puis s'inscrire en master en France. L'option d'un retour aux Comores est également évoquée, si ses frères l'aident à y monter un projet entrepreneurial. Pendant la première année à Dakar, l'appui financier de ses frères est essentiel. Le troisième lui a payé le billet d'avion pour Dakar, le cadet prend en charge son inscription dans un institut privé et lui envoie de quoi vivre et l'aîné, inscrit en doctorat et dont les revenus sont faibles et irréguliers, l'appuie en cas de problème. La deuxième année, le troisième quitte son poste d'ingénieur pour créer son entreprise et, n'ayant plus de revenus fixes, ne finance plus Naïm. Les deux autres prennent le relais. À la fin de la troisième année, le cadet se lance dans la préparation de son mariage et demande à l'aîné, doctorant, de prendre seul en charge les études de Naïm, ce qu'il ne parvient pas à faire. Devant ses frères, Naïm se montre compréhensif : "J'ai dit bon, c'est mieux de rentrer parce qu'au moins je suis chez moi, je vais pas payer de loyer, je vais pas payer quoi que ce soit. [...] Vous avez fait ce que vous avez pu, y’a pas de souci. » Mais au cours des entretiens répétés, il se montre amer face à ceux qui lui avaient promis un soutien continu et l'ont finalement contraint à rentrer aux Comores, sans l'aider à mettre en place le projet professionnel dont il était question au départ. Il se montre particulièrement critique vis-à-vis du cadet, qui avait encouragé l'aîné et Naïm à poursuivre leurs études en leur garantissant son appui, et qui a préféré dépenser plus de 4000 euros pour son mariage, c'est-à-dire " pour rien » (Naïm H., 26 ans, émigré de retour comorien, master de commerce, sans emploi).

L'exemple de Naïm montre que, dans les familles dispersées, dont les revenus sont faibles ou irréguliers et dans lesquelles l'entraide ne peut être garantie dans la durée, essayer de faire partir l'un des membres dans une destination intermédiaire, en attendant une amélioration financière facilitant un départ plus lointain, reste une trajectoire à l'issue très incertaine. Selon les ressources contrastées dont ils disposent, les uns renoncent à leur désir de mobilité ou renégocient leur destination, quand les autres essayent de s'accommoder de la frontière française en la franchissant par étapes.

\section{Contourner la frontière}

La dernière possibilité pour les étudiants comoriens et togolais consiste à contourner la frontière française, en choisissant des destinations jugées "plus faciles d'accès». Ainsi, l'Allemagne, les États-Unis ou le Canada sont des 
destinations régulièrement évoquées au Togo ${ }^{49}$, le Royaume-Uni ou les pays du Golfe l'étant plus souvent aux Comores. Si ces lieux d'émigration sont marginalement évoqués comme des solutions de secours par les étudiants populaires diplômés qui anticipent leurs futures difficultés à émigrer en France, ils sont plus clairement envisagés dans les fractions économiques des catégories supérieures (commerçants, cadres du secteur privé, chefs d'entreprise et professions libérales). Les pays anglo-saxons sont devenus des lieux d'émigration distinctifs, investis d'un certain prestige dans l'imaginaire cosmopolite des élites socioéconomiques africaines. Ces nouvelles destinations sont valorisées, notamment parce qu'elles sont, selon les enquêtés, marquées par une ouverture culturelle et un dynamisme qui feraient défaut à la France. Mais le succès de ces contournements dépend une nouvelle fois des ressources individuelles et familiales. Avant de chercher à initier ces pratiques spatiales d'adaptation aux politiques migratoires françaises, il faut avoir été directement disposé par son entourage à cette géographie migratoire remodelée. Ces savoir-faire migratoires inégaux sont transmis par des parents ou des amis qui aiguillent les demandeurs. Dans les milieux populaires, les étudiants sont assez peu directement confrontés à ces expériences migratoires prestigieuses au sein de leur entourage, ce qui rend ces réorientations spatiales peu envisageables et matériellement peu probables. Certains de ces émigrés, notamment togolais, ont pu partir vers ces destinations grâce aux bourses étudiantes obtenues avant les années 1990, mais ces itinéraires sont désormais moins courants.

En étudiant les conditions d'accès aux différents pays d'accueil, on comprend que si les franges les plus diplômées des milieux populaires valorisent ces nouvelles destinations, au même titre que les émigrés socialement favorisés, cela ne signifie pas la fin des frontières entre ces groupes sociaux. Ce qui les distingue réside dans le plus ou moins grand accès à ces destinations. Il existe bel et bien une homologie entre la structuration de l'espace social et la hiérarchie symbolique des destinations effectivement accessibles. Comme l'avait souligné Nicholas Van Hear, un émigré part souvent vers " la destination qu'il peut atteindre avec l'argent et les relations dont il dispose ${ }^{50} »$. Ce constat souligne la forte dimension spatiale des inégalités sociales, que renforce la fermeture des frontières françaises. Les populations qui disposent de faibles ressources sont plus souvent contraintes de vivre dans des « espaces dévalorisés ${ }^{51} »$.

49. La référence nord-américaine s’observe également au Mali. Mary (K.), "Entre la "forteresse Europe" et l'Amérique d'Obama. Le choix des États-Unis pour les ressortissants maliens ", Hommes et Migrations, 1286-1287, 2010.

50. Van Hear (N.), « Reconsidering Migration and Class », International Migration Review, 48, 2014, p. 101.

51. Backouche (I.) et al., La dimension spatiale des inégalités. Regards croisés des sciences sociales, Rennes, Presses universitaires de Rennes, 2011, p. 272. 


\section{Conclusion}

Loin des discours célébrant le cosmopolitisme de citoyens qualifiés, les étudiants internationaux africains ne constituent pas une catégorie à part, mais demeurent des étrangers parmi les autres, soumis à un contrôle politique prégnant, à l'œuvre dès les consulats. Les étudiants comoriens et togolais doivent faire face aux représentations sociales dépréciatives des agents consulaires chargés d'autoriser leurs déplacements. La comparaison entre ces deux cas nationaux démontre que ces assignations identitaires ne sont certes pas homogènes. Il n'en reste pas moins que, depuis près de quarante ans, ces étudiants subissent un double rejet, à la fois stigmatisés en tant qu'étrangers, dont les frontières françaises limitent les départs et l'installation en France, et en tant qu'étudiants dont les dossiers sont hiérarchisés à l'aune de critères utilitaristes. En comparant les pays d'origine et les propriétés sociales des demandeurs, on comprend que les politiques consulaires de délivrance du visa étudiant n'ont pas sensiblement réduit les inclinations à la mobilité, puisqu'elles s'avèrent aujourd'hui plus sélectives que pleinement restrictives ${ }^{52}$.

Renverser la perspective, en analysant ces mobilités internationales depuis les parcours des étudiants, offre de nouveaux éclairages sur ces logiques frontalières. Comme les autres émigrés, ces étudiants doivent obtenir un visa dont l'acquisition nécessite la possession d'importantes ressources matérielles ${ }^{53}$. Mais la gestion des demandes par les services culturels des ambassades, puis l'institutionnalisation à partir de 2010 des espaces Campus France, ont renforcé la nécessité de détenir des ressources immatérielles (scolaires, culturelles, linguistiques et symboliques). En toute logique, les catégories sociales les plus aisées sont les premières à s'approprier ces démarches, quand ce dispositif frontalier capte les demandeurs issus de milieux sociaux plus précaires. Mais cet article démontre surtout qu'il existe des modes d'émigration étudiante différenciés. D'un côté, les fractions basses des catégories supérieures sont plus aptes à mobiliser des savoir-faire, des savoir-être et une «bonne volonté administrative ${ }^{54}$ » et ainsi à répondre aux exigences consulaires; de l'autre, les fractions hautes des catégories populaires jouent sur des ressources diversifiées liées à la dispersion intergénérationnelle de leur famille et sur des formes de " débrouille ${ }^{55}$ ", pour s'ajuster et/ou contourner cette frontière française légale. Cette mobilité internationale apparaît donc tour à tour " "acquise", "conquise"

\footnotetext{
52. Bonjour (S.), Chauvin (S.), « Social Class, Migration Policy and Migrant Strategies: An Introduction ", International Migration, 56 (4) 2018 ; de Haas (H.) et al., « Growing Restrictiveness or Changing Selection? The Nature and Evolution of Migration Policies », International Migration Review, 2016.

53. Bréant $(\mathrm{H}$.$) , « (\mathrm{Im})$ mobilité internationale : les inégalités au sein des catégories populaires face à la migration ", Lien social et politiques, 74, 2015.

54. Dubois (V.), La vie au guichet, op. cit.

55. Siblot (Y.), Faire valoir ses droits au quotidien, op. cit.
} 
ou "entravée" ${ }^{56}$ ", au regard des propriétés sociales et des histoires familiales contrastées de ces étudiants africains. Le succès des entreprises étudiantes ne devient dès lors pleinement lisible qu'au prisme de ces dynamiques collectives et intergénérationnelles.

Alors que les catégories populaires sont maintenues sous le contrôle permanent des politiques d'immigration ${ }^{57}$, les étudiants issus de ces milieux sociaux parviennent régulièrement à contourner les frontières, mais au prix de mobilités spatiales moins linéaires, constamment complexifiées ${ }^{58}$, souvent bloquées et aux temporalités plus longues. Comme les autres émigrés, ces étudiants mettent en ouvre des « jeux possibles avec l'instrument » consulaire ${ }^{59}$. Mais l'obstacle du visa étudiant s'avère être un outil politique contraignant qui laisse peu de place à la négociation et aux accommodements. Dès lors, les frontières légales ont tendance à renforcer les inégalités sociales des pays d'origine ${ }^{60}$.

\begin{abstract}
Hugo BréANt est post-doctorant à I'Université de Rouen Normandie (Dysolab) et docteur en science politique de I'Université Paris 1 (CESSP). II est affilié à I'IC Migrations (2018-2021). Ses recherches interrogent l'articulation entre mobilités spatiales et mobilités sociales dans les parcours biographiques d'émigrés comoriens et togolais. En analysant les dispositions, les représentations et les pratiques des individus, son travail montre comment les migrations favorisent tout à la fois la reproduction des inégalités et le brouillage des frontières sociales dans les pays d'origine
\end{abstract}

(" (Im)mobilité internationale: les inégalités au sein des catégories populaires face à la migration ", Lien social et politiques, $n^{\circ} 74,2015$, p. 37-56). Sa thèse comparative, intitulée "Les chemins internationaux de la mobilité sociale ", a reçu en 2017 le prix de la Commission de la recherche de I'Université Paris 1. II a récemment coordonné, avec Sébastien Chauvin et Ana Portilla, un numéro de la revue Actes de la recherche en sciences sociales consacré au "Capital social en migration » (à paraître en décembre 2018).

\footnotetext{
56. Gérard (É.), dir., Mobilités étudiantes Sud-Nord. Trajectoires scolaires de Marocains en France et insertion professionnelle au Maroc, Paris, Publisud, 2008, p. 24.

57. Fischer (N.), « Les expulsés inexpulsables. Recompositions du contrôle des étrangers dans la France des années 1930 », Cultures \& Conflits, 53, 2004.

58. Ngwé (L.), «Globalisation et mobilités internationales des étudiants au Cameroun », Hommes \& Migrations, 1307, 2014.

59. Barrault-Stella (L.), « Jouer avec l'instrument. Les usages institutionnels et sociaux de la carte scolaire ", in Halpern (C.) et al., dir., L'instrumentation de l'action publique. Controverses, résistance, effets, Paris, Presses de Sciences Po, 2014, p. 412.

60. Bréant (H.), «Les chemins internationaux de la mobilité sociale. Expériences de mobilité et d'immobilité sociales dans les parcours migratoires comparés d'émigrés comoriens et togolais ", thèse de science politique, Paris 1, 2016, $743 \mathrm{p}$.
} 
African students: Migrants like the others.

Social selectivity of the visa and spatial (im)mobilities of Comorian and Togolese international students

Although political discourses emphasize the attractiveness of France and the reception conditions for foreign students, these students remain strangers like any others, progressively facing monitoring policies over their entry and stay in France. African students are at the forefront of this border closure, implemented from the consulates. This article proposes to reverse the perspective by observing not the production of these borders, but the perceptions and practices of the students who face them. Investigating students' life courses-both those who have obtained their visa and those who have not-reveals the inequalities introduced by this political filter, which is very socially selective. Often confined by a student visa procedure, students develop different strategies to adapt to borders, or to bypass them, according to their social profile, their family history, and their individual and collective resources. This approach to public policies from below, and from its users, explains how the inextricable imbrication of borders, both political and social, reinforces the spatial immobility of some, but above all makes the mobility of others more complex.

Keywords - international mobility, students, borders, visas, social inequalities, Comoros, Togo 